\title{
The curvature of the universe and the dark energy potential
}

\author{
Sergio del Campo ${ }^{a}$ \\ a Instituto de Física, Universidad Católica de Valparaíso, \\ Casilla 4059, Valparaíso, Chile.
}

(Dated: November 30, 2018)

\begin{abstract}
The flatness of an accelerating universe model (characterized by a dark energy scalar field $\chi$ ) is mimicked from a curved model that is filled with, apart from the Cold Dark Matter component, a quintessence-like scalar field $Q$. In this process we characterize the original scalar potential $V(Q)$ and the mimicked scalar potential $V(\chi)$ associated to the scalar fields $Q$ and $\chi$, respectively. The parameters of the original model are fixed through the mimicked quantities that we relate to the present astronomical data, such that the equation state parameter, $w_{\chi}$ and the dark energy density parameter, $\Omega_{\chi}$

PACS numbers: $98.80 . \mathrm{Hw}, 98.80 . \mathrm{Bp}$
\end{abstract}

\section{INTRODUCTION}

At the moment, we do not precisely know the amount of matter present in the universe, but we do not as yet know what the geometry of the universe is. Astronomical observations conclude that the matter density related to baryonic and nonbaryonic cold dark matter is much less than the critical density value [1]. However, measurements of anisotropy in the cosmic microwave background radiation indicate that the total matter present in the universe is very much the same than its critical value [2, 3, 4, 5]. These measurements agree with the theoretic prediction of inflationary universe scenarios [6], which predicts that our universe should become flat after leaving the inflationary era. In light of these results, it seems that there exist an important amount of matter that we are not considering.

On the other hand, recent measurements of type Ia supernova at high redshift indicate that our universe is accelerating [7. 8] . The most simple description of this acceleration is that it can be characterized as a cosmological constant, which contributes to a negative pressure. Other approaches have been employed for explaining this acceleration. We distinguish that related to the quintessence model. This model is characterized by an evolving scalar field, $\chi$ and its scalar-field potential, $V(\chi)$ [9].

Any cosmological model becomes characterized by the total matter density parameter, $\Omega_{T}$. This parameter is defined by the ratio between the total matter $\left(\rho_{T}\right)$ and the critical energy $\left(\rho_{C}\right)$ densities. For instance, in the case of the Cosmological Constant $(\Lambda)$ Cold Dark Matter $(\mathrm{CDM})$ model, this parameter is given by $\Omega_{T}=$ $\Omega_{M}+\Omega_{\Lambda}$, in which $\Omega_{M}=\frac{\rho_{M}^{0}}{\rho_{C}}=\left(\frac{8 \pi G}{3 H_{0}^{2}}\right) \rho_{M}^{0}$ and $\Omega_{\Lambda}=\left(\frac{\Lambda}{8 \pi G}\right) \frac{1}{\rho_{C}}=\frac{\Lambda}{3 H_{0}^{2}}$. Here, $\rho_{M}^{0}$ is the actual value of the nonrelativistic matter density and $H_{0}$, the actual value of the Hubble parameter. $\Omega_{\Lambda}$ is the fraction of the critical energy density contained in a smoothly distributed vacuum energy referred to as $\Lambda$, and $\Omega_{M}$ represents the matter density related to the baryonic and non- baryonic CDM densities. The constant $G$ is the Newton constant, and we use $c=1$ for the speed of light. From now on, all quantities with upper or lower zero indexes specify current values.

Due to measurements and theoretical arguments, it seems natural to consider flat universe models, but one interesting question to ask is whether this flatness is due to a sort of compensation among different components that enter into the dynamical equations. In this respect, our main goal in this paper is to address this sort of question. In the literature we have found some descriptions along these lines. For instance, closed models with an important matter component with equation of state given by $P=-\rho / 3$ have been studied. Here, the universe expands at constant speed [10]. Other authors, by using the same properties for the universe, have added a nonrelativistic matter density in which $\Omega_{T}$ is less than one, thus describing an open universe 11. Also, flat decelerating models have been simulated [12]. The common fact in all of these models is that, even if the starting geometry presents curvature, all models are indistinguishable from flat geometries at low redshift.

In this paper we wish to consider universe models that have curvature and are composed by two-matter components. One of these components is the usual nonrelativistic dust matter; the other corresponds to a sort of quintessence-type matter, described by a scalar field that we designate by $Q$. This field is fundamental in the sense that it is introduced from a Lagrangian. In order to mimic a flat Quintessence CDM, we introduce a new scalar field $\chi$, such that this field is constrained by recent astronomical data. Therefore, in a flat background the scalar field $\chi$ together with the CDM component form the basis for the $\chi \mathrm{CDM}$ model, a model that is restricted by the present observations. In this way, it is the scalar field $\chi$, not the $Q$-field, that is related to the observable quantities [13]. Thus, we assume that the effective equation of state for $\chi$ is given by $P_{\chi}=w_{\chi} \rho_{\chi}$, where $w_{\chi}$ is the observable effective equation state parameter [9]. We note that the astronomical observations (related to the type Ia supernovae measurements) put an upper limit on the present value of this parameter, $w_{\chi}<-1 / 3[?]$. 
We also assume that the scalar field $Q$ is characterized by a similar equation of state

$$
P_{Q}=w_{Q} \rho_{Q},
$$

where the parameter $w_{Q}$ is, in general, a variable quantity. Therefore, our goal in this paper is to investigate the conditions under which a scenario with positive (or negative) curvature may mimic a flat universe at low redshifts. This approach forces us to determine the exact contribution of the scalar field $Q$, together with the curvature term, that gives rise to the flat quintessence accelerating $\chi \mathrm{CDM}$ universe. However, we should comment that absorbing the curvature term in a redefinition of the $Q$ field is certainly not equivalent to getting a really flat universe, since the curvature is a geometrical property, which follows directly from the metric tensor and which enters into the FRW line element. As we mentioned above, these two models become indistinguishable only for low redshifts and are similar to the cases studied in ref. [11, 12]. We will return to this point later on. Here, we emphasize that our approach rests in the fact that there is a clear similitude between the curvature and the flat universe models at low redshift.

\section{THE DYNAMIC FIELD EQUATIONS}

We start with the following effective action

$$
\begin{aligned}
S=\int d^{4} x \sqrt{-g}[ & \frac{1}{16 \pi G} R \\
& \left.+\frac{1}{2}\left(\partial_{\mu} Q\right)^{2}-V(Q)+L_{M}\right],
\end{aligned}
$$

where $R$ is the scalar curvature, $V(Q)$ is the scalar potential associated to the field $Q$ and $L_{M}$ is related to any ordinary matter component.

We shall assume that the $Q$ field is homogeneous, i.e. it is a time-depending quantity only, $Q=Q(t)$, and the spacetime is isotropic and homogeneous, with metric corresponding to the Friedman-Robertson-Walker metric

$$
\begin{aligned}
d s^{2}=d t^{2}-a(t)^{2} & {\left[\frac{d r^{2}}{1-k r^{2}}\right.} \\
& \left.+r^{2}\left(d \theta^{2}+\sin ^{2} \theta d \phi^{2}\right)\right],
\end{aligned}
$$

where $a(t)$ represents the scale factor and the parameter $k$ takes the values $k=-1,0,1$ corresponding to an open, flat, closed three-geometry, respectively. With these assumptions, the action (2) yields the following field equations. The time-time component of Einstein equation

$$
H^{2}=\frac{8 \pi G}{3}\left(\rho_{M}+\rho_{Q}\right)-\frac{k}{a^{2}}
$$

the evolution equation for the scalar field $Q$

$$
\ddot{Q}+3 H \dot{Q}=-\frac{\partial V(Q)}{\partial Q} .
$$

and the energy conservation law for the ordinary matter

$$
\dot{\rho}_{M}+3 H\left(\rho_{M}+P_{M}\right)=0 .
$$

In these Equations the overdots denote derivatives with respect to $t, H=\frac{\dot{a}}{a}$ defines the Hubble expansion rate, $\rho_{M}$ and $\rho_{Q}$ are the effective matter energy density and the average energy densities, respectively. The Q-energy density is defined by

$$
\rho_{Q}=\frac{1}{2} \dot{Q}^{2}+V(Q) .
$$

We introduce its average pressure $P_{Q}$, by means of

$$
P_{Q}=\frac{1}{2} \dot{Q}^{2}-V(Q) .
$$

These two latter quantities are related by the equation of state, Eq. (11). Thus, expression (5) becomes

$$
\dot{\rho}_{Q}+3 H\left(\rho_{Q}+P_{Q}\right)=0,
$$

which, similar to the ordinary matter, represents an energy balance for the scalar field $Q$. From now on, we consider the ordinary matter to correspond to dust, which becomes characterized by the equation of state $P_{u}=0$. For this case we could solve the energy Equation 6 analytically, in which case we get $\rho_{M} \propto a^{-3}$.

Summarizing, we have a combination of two noninteracting perfect fluids : one, a dust matter component, $\left(\rho_{M}\right)$; the other, the scalar field $\left(\rho_{Q}\right)$ component.

Eq. (㺼) may be written as

$$
\begin{aligned}
H^{2}=H_{0}^{2}\left[\Omega_{M}\right. & \left(\frac{\rho_{M}}{\rho_{M}^{0}}\right) \\
& \left.+\Omega_{Q}\left(\frac{\rho_{Q}}{\rho_{Q}^{0}}\right)+\Omega_{k}\left(\frac{a_{0}}{a}\right)^{2}\right] .
\end{aligned}
$$

Here, the actual curvature density, $\Omega_{k}$, and the quintessence density, $\Omega_{Q}$, parameters are defined by $\Omega_{k}=-k\left(\frac{1}{a_{0} H_{0}}\right)^{2}$, and $\Omega_{Q}=\left(\frac{8 \pi G}{3 H_{0}^{2}}\right) \rho_{Q}^{0}$, respectively.

In the next section we study the model that arises when the matter component, $\rho_{M}$ together with Eqs. (4), (5) and the equation of state for the field $Q$, complement in such a way that a flat CDM accelerated universe originates, specifically, the $\chi \mathrm{CDM}$ model.

In order to simulate a flat universe, we assume that the energy density $\rho_{Q}$ and the curvature term combine so that

$$
\frac{8 \pi G}{3} \rho_{Q}(t)-\frac{k}{a^{2}(t)}=\frac{8 \pi G}{3} \rho_{\chi}(t),
$$


or equivalently,

$$
\Omega_{Q}\left(\frac{\rho_{Q}}{\rho_{Q}^{0}}\right)+\Omega_{k}\left(\frac{a_{0}}{a}\right)^{2}=\Omega_{\chi}\left(\frac{\rho_{\chi}}{\rho_{\chi}^{0}}\right),
$$

where, similar to the definitions of $\Omega_{M}$ and $\Omega_{Q}$, we have defined $\Omega_{\chi}=\left(\frac{8 \pi G}{3 H_{0}^{2}}\right) \rho_{\chi}^{0}$. This latter quantity is related to the recent astronomical measurement of distant supernova of type Ia.

Note that we could get an explicit expression (as a function of time) for the unknown energy density, $\rho_{\chi}$, if we know both the scale factor $a(t)$ and the time dependence of the $Q$-density, $\rho_{Q}$. Note also that for $\Omega_{k}>0$ (open universes) we must have $\rho_{\chi}>\frac{\left|\Omega_{k}\right|}{\Omega_{\chi}} \rho_{\chi}^{0}\left(\frac{a_{0}}{a}\right)^{2}$, since $\rho_{Q}>0$. At this point we should comment on the difference between the two scalar fields $Q$ and $\chi$. As we saw, the scalar field $Q$ is defined from the fundamental Lagrangian, from which we could define the stress-energy density tensor with the property of a perfect fluid behaviour, which allows us to introduce the pressure $P_{Q}$. However, we could not say the same of $\chi$. The definition of the stress-energy density tensor associated with the $\chi$ field is more subtle, as we can see in Eq. (11) (or (12)). We hope to address this problem in subsequent work. Here, we just take $P_{\chi}$ as an effective pressure that follows the equation of state $P_{\chi}=w_{\chi} \rho_{\chi}$, where $w_{\chi}$ is determined from the observational data.

\section{CHARACTERISTICS OF THE MODEL}

In this section we will impose explicit conditions under which a curved universe $(k= \pm 1)$ may look similar to a flat universe $(k=0)$ at low redshift. This flat model is defined by expression (11) (or (12)), which reduces the time-time Einstein Eq. (10) to

$$
H^{2}=H_{0}^{2}\left[\Omega_{M}\left(\frac{\rho_{M}}{\rho_{M}^{0}}\right)+\Omega_{\chi}\left(\frac{\rho_{\chi}}{\rho_{\chi}^{0}}\right)\right] .
$$

Eqs. (12) and (13), along with the evolution equations for the scalar fields $\chi$ and $Q$, form the basic set of equations that describes our model.

Note that, when Eq. 12 is evaluated at present time, we obtain the following relation among the omega parameters

$$
\Omega_{Q}=\Omega_{\chi}-\Omega_{k} .
$$

From this relation we observe that, for $k=1, \Omega_{Q}$ must be greater than $\Omega_{\chi}$, since $\Omega_{k=1}<0$. For $k=-1, \Omega_{k}>$ 0 , thus $\Omega_{\chi}>\Omega_{Q}$. Notice also that Eq. (13) gives the additional expression $\Omega_{M}+\Omega_{\chi} \equiv \Omega_{T}=1$ when evaluated at present time. From the present observational values of $\Omega_{\chi}$ and $\Omega_{k}$, we could get the actual value of the parameter $\Omega_{Q}$. Note also that when Eqs. (10) and (13) are compared we observe that the variables $\rho_{M}$ and $H$ (and also the scale factor $a$, as we can see from Eq. (11) or equivalently Eq. (12)) appear identical in the two scenarios.

In order to see that a curvature model at low redshift is indistinguishable from a flat one, we could consider the luminosity distance $D_{L}$ as a function of the redshift $z$ or the angular distance 12]. Let us take the first one. The luminosity distance between a source at a redshift $z>0$ and $z=0$, related to a curvature model is obtained from the expression

$$
D_{L}^{k \neq 0}(z) \propto \frac{(1+z)}{\sqrt{\left|\Omega_{k}\right|}} \sin \left[\sqrt{\left|\Omega_{k}\right|} \xi(z)\right] .
$$

For a flat universe model we obtain

$$
D_{L}^{k=0}(z) \propto(1+z) \xi(z) .
$$

In these expressions we have defined $\xi(z)$ by means of $\xi(z)=\frac{1}{a_{0}} \int_{0}^{z} \frac{d z}{H(z)}$ which represents the polarcoordinated distance between a source at $z$ and another at $z=0$ in the same line of sight. For $z \ll 1$ we could show that $\xi(z) \propto z$. Thus, since $\left|\Omega_{k}\right| \ll 1$ we observe that the luminosity distance in both cases coincide. Therefore, we expect that the differences between the curvature and the flat models happen to high enough redshifts.

When the quintessence component has a constant equation state parameter, $w_{\chi}^{0} \equiv w_{\chi}$, which is a negative number that lies in the range $-1<w_{\chi}<-0.3$, we could immediately solve for the density $\rho_{\chi}$ as a function of the scale factor

$$
\rho_{\chi}(a)=\rho_{C} \Omega_{\chi}\left(\frac{a_{0}}{a}\right)^{3\left(1+w_{\chi}\right)} .
$$

Thus, Eq. (13) becomes

$$
H(a)=H_{0}\left(\frac{a_{0}}{a}\right)^{3 / 2} \sqrt{\Omega_{M}+\Omega_{\chi}\left(\frac{a_{0}}{a}\right)^{3 w_{\chi}}} .
$$

The solution of Eq. 18) is given by

$$
\begin{aligned}
& t=\frac{2}{3 H_{0} \sqrt{\Omega_{M}}}\left(\frac{a}{a_{0}}\right)^{3 / 2} \\
& \times{ }_{2} F_{1}\left(\frac{1}{2},-\frac{1}{2 w_{\chi}} ; 1-\frac{1}{2 w_{\chi}} ;-\left(\frac{\Omega_{k}+\Omega_{Q}}{\Omega_{M}}\right)\left(\frac{a}{a_{0}}\right)^{-3 w_{\chi}}\right),
\end{aligned}
$$

where ${ }_{2} F_{1}$ represents the generalized hypergeometric function. The initial condition that we have used in solving Eq. (18) is $a=0$ at $t=0$.

We use the definition of $P_{\chi}$ and $\rho_{\chi}$ in terms of the scalar field $\chi$, together with the equation of state that relates these quantities, for obtaining $\chi$ as a function of the scale factor. The result is

$$
\chi(a)=\chi_{0}\left(\frac{a}{a_{0}}\right)^{-3 w_{\chi} / 2}
$$




$$
\times\left[\frac{{ }_{2} F_{1}\left(\frac{1}{2}, \frac{1}{2} ; \frac{3}{2} ;-\left(\frac{\Omega_{k}+\Omega_{Q}}{\Omega_{M}}\right)\left(\frac{a}{a_{0}}\right)^{-3 w_{\chi}}\right)}{{ }_{2} F_{1}\left(\frac{1}{2}, \frac{1}{2} ; \frac{3}{2} ;-\left(\frac{\Omega_{k}+\Omega_{Q}}{\Omega_{M}}\right)\right)}\right],
$$

where $\chi_{0}$ is given by $\chi_{0}=$ $\tilde{\chi}_{2} F_{1}\left(\frac{1}{2}, \frac{1}{2} ; \frac{3}{2} ;-\left(\frac{\Omega_{k}+\Omega_{Q}}{\Omega_{M}}\right)\right)$, with $\tilde{\chi}=$ $\sqrt{4 \rho_{c} / 9 H_{0}^{2}} \sqrt{\left(\Omega_{k}+\Omega_{Q}\right)\left(1+w_{\chi}\right) / \Omega_{M} w_{\chi}^{2}}$.

In a similar way, we get for the scalar potential $V_{\chi}$

$$
V_{\chi}(a)=V_{\chi}^{0}\left(\frac{a_{0}}{a}\right)^{3\left(1+w_{\chi}\right)},
$$

where $V_{\chi}^{0}=\frac{1}{2}\left(1-w_{\chi}\right) \rho_{C}\left(\Omega_{k}+\omega_{Q}\right)$.

Fig (11) shows the plot of the scalar potential $V_{\chi}$ as a function of $\chi$, for three different values of the equation state parameter $w_{\chi}$. The parameters $\Omega_{\chi}\left(=\Omega_{k}+\Omega_{Q}\right)$ and $\Omega_{M}$ have been fixed at values 0.65 and 0.35 , respectively. This form of potential has been described in the literature 14]. Note that, in the limit $w_{x} \longrightarrow-1$ the po-

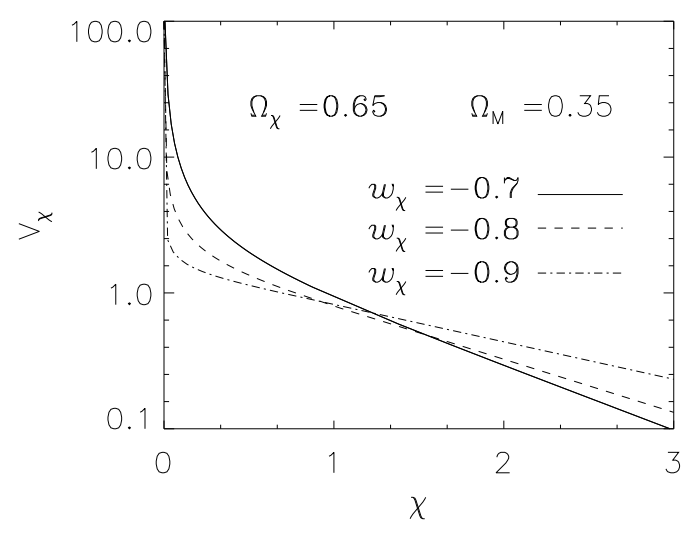

FIG. 1: This graph shows the scalar potential $V_{\chi}$ as a function of the scalar field $\chi$ for $w_{\chi}=-0.7,-0.8$ and -0.9 . We use $\Omega_{M}=0.35$ and $\Omega_{\chi}=0.65$.

tential $V_{\chi} \longrightarrow$ const. $\equiv \rho_{C} \Omega_{\chi}$, i.e., the model becomes equivalent to the $\Lambda$ Cold Dark Matter scenario 9 .

During the evolution of this model, we distinguish two periods: one, where the regular matter dominates $\left(\rho_{M} \gg\right.$ $\left.\rho_{\chi}\right)$; the other, where $\rho_{\chi} \gg \rho_{M}$. It is not hard to see that the time at which $\rho_{M}$ becomes equal to $\rho_{\chi}$ is given by

$$
\begin{aligned}
t_{e q}= & \frac{2}{3 H_{0} \sqrt{\Omega_{M}}}\left(\frac{\Omega_{M}}{\Omega_{\chi}}\right)^{-\frac{1}{2 w_{\chi}}} \\
& \quad{ }_{2} F_{1}\left(\frac{1}{2},-\frac{1}{2 w_{\chi}} ; 1-\frac{1}{2 w_{\chi}} ;-1\right) .
\end{aligned}
$$

Notice that the age of the universe in this model is given by

$$
\begin{aligned}
& t_{0}=\frac{2}{3 H_{0} \sqrt{\Omega_{M}}} \\
& \times{ }_{2} F_{1}\left(\frac{1}{2},-\frac{1}{2 w_{\chi}} ; 1-\frac{1}{2 w_{\chi}} ;-\frac{\Omega_{\chi}}{\Omega_{M}}\right),
\end{aligned}
$$

where, similar to $t_{e q}$, depends on the observable param-

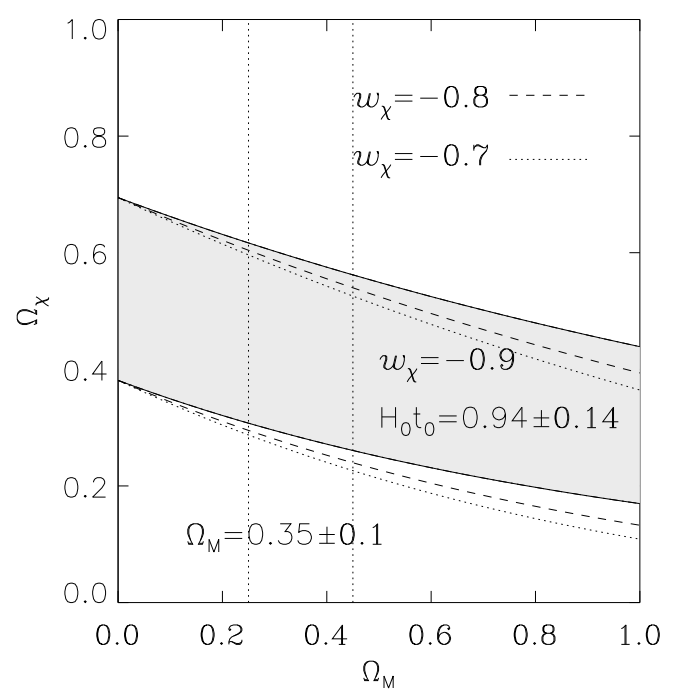

FIG. 2: This graph represents $\Omega_{\chi}$ as a function of $\Omega_{M}$ for the range of $t_{0} H_{0}=0.94 \pm 0.14$ for three different values of the equation state parameter, $w_{\chi}=-0.7,-0.8,-0.9$. The dark region corresponds to $w_{\chi}=-0.9$. The vertical dotted lines show the observational range for the $\Omega_{M}$ parameter.

eters $\Omega_{\chi}, \Omega_{M}$ and $w_{\chi}$. For $\Omega_{M}=0.3, \Omega_{\chi}=0.7$ and $w_{\chi}=-0.8$ this gives $t_{0} H_{0} \sim 0.93$, which lies in the observational range $t_{0} H_{0}=0.94 \pm 0.14$ of the measurement of the age of the universe [15]. Fig (2) shows $\Omega_{\chi}$ as a function of $\Omega_{M}$ for the range of $t_{0} H_{0}$ specified above. The dark region represents the value $w_{\chi}=-0.9$.

We are now going to describe the properties of the scalar field $Q$. Following an approach analogous to that used for the scalar field $\chi$, we find that the parameter $w_{Q}$, is given by

$$
\begin{aligned}
w_{Q}(a)=- & \left|w_{Q}^{0}\right|\left(\frac{1+\beta}{1-3 \beta w_{\chi}}\right) \\
& \times\left[\frac{1-3 \beta w_{\chi}\left(\frac{a}{a_{0}}\right)^{-3 w_{\chi}-1}}{1+\beta\left(\frac{a}{a_{0}}\right)^{-3 w_{\chi}-1}}\right],
\end{aligned}
$$

where $\beta=\Omega_{\chi} /\left|\Omega_{k}\right|$ and $w_{Q}^{0}$ is the actual value of $w_{Q}$ defined by $w_{Q}^{0}=P_{Q}^{0} / \rho_{Q}^{0}$. Fig (3) shows its dependence 
with the redshift $z$ defined as $z \equiv a_{0} / a-1$, for three different values of the $w_{\chi}$ parameter. For completeness, we have chosen $\Omega_{Q}=0.85$ and $\Omega_{\chi}=0.65$ in this plot, i.e. we have considered a closed model.

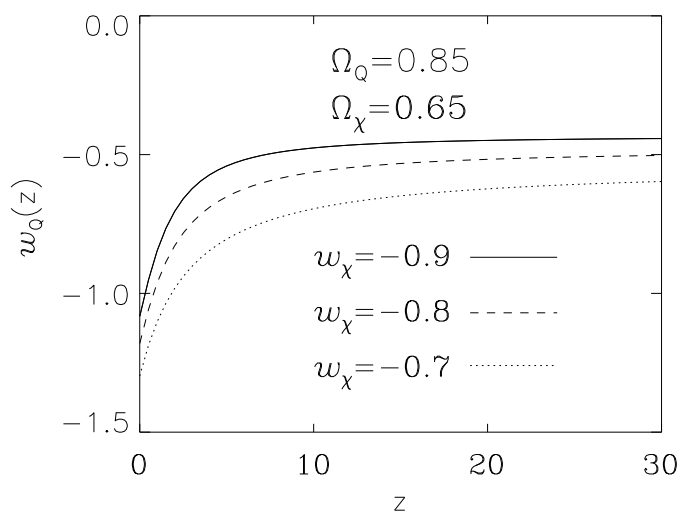

FIG. 3: The graph shows $w_{Q}$ (in unit of $\left|w_{Q}^{0}\right|$ ) as a function of the redshift $z$ for three different values of the equation state parameter, $w_{\chi}=-0.7,-0.8,-0.9$. We have used the values $\Omega_{Q}=0.85$ and $\Omega_{\chi}=0.65$, corresponding to a closed model.

The scalar field $Q$ results in

$$
Q(a)=\bar{Q} \int_{0}^{a / a_{0}} \sqrt{\frac{1+\frac{3}{2} \beta\left(1+w_{\chi}\right) x^{-\left(1+3 w_{\chi}\right)}}{x+\frac{\Omega_{\chi}}{\Omega_{M}} x^{-\left(1+3 w_{\chi}\right)}}} d x,
$$

where $\bar{Q}=\sqrt{\frac{1}{4 \pi G}\left(\frac{\left|\Omega_{k}\right|}{\Omega_{M}}\right)}$.

The scalar potential, $V_{Q}(a)$, is found to be given by

$$
\begin{aligned}
V_{Q}(a)= & V_{Q}^{0}\left(\frac{a_{0}}{a}\right)^{2} \\
& \times\left[\frac{4+3 \beta\left(1-w_{\chi}\right)\left(\frac{a}{a_{0}}\right)^{-\left(1+3 w_{\chi}\right)}}{4+3 \beta\left(1-w_{\chi}\right)}\right],
\end{aligned}
$$

where $V_{Q}^{0}=\frac{\rho_{C} \Omega_{Q}}{2\left(1-w_{\chi}\right)}\left[4 / 3+\beta\left(1-w_{\chi}\right)\right]$. Figure (14) shows the potential $V_{Q}$ as a function of the scalar field $Q$ for three different values of $w_{\chi}$. Again, we have considered a closed model with $\Omega_{Q}=0.85, \Omega_{M}=0.35$ and $\Omega_{\chi}=0.65$. For open models, i.e. for $\Omega_{\chi}>\Omega_{Q}$ these curves are very similar.

Note that the potential decreases when $Q$ increases. We observe that this potential asymptotically tends to vanish for $a \longrightarrow \infty$. This implies that, asymptotically, the effective equation of state becomes $P_{Q}=\rho_{Q}$ (for $\dot{Q} \neq 0$ ), corresponding to a stiff fluid.

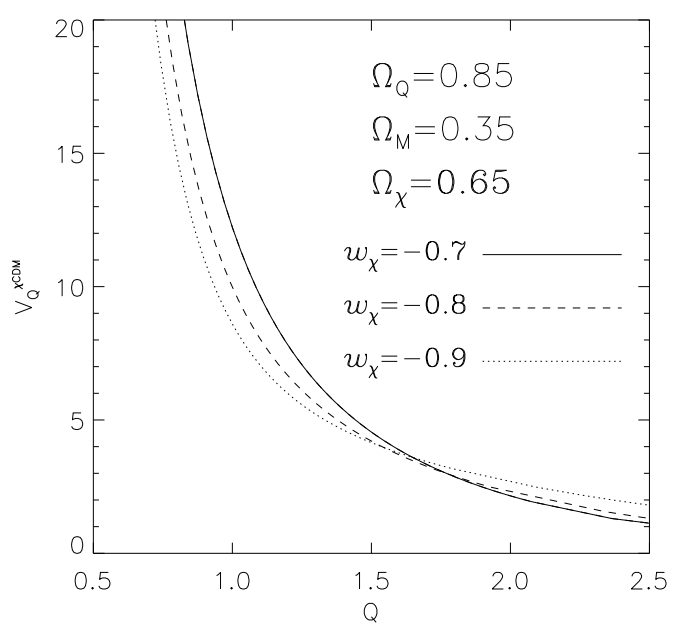

FIG. 4: The graph shows $V_{Q}$ (in unit of $\rho_{C} / 2$ ) as a function of $Q$ (in unit of $1 / \sqrt{4 \pi G}$ ) for three different values $w_{\chi}=$ $-0.7,-0.8$, and -0.9 . We use the values $\Omega_{Q}=0.85, \Omega_{\chi}=$ 0.65 and $\Omega_{M}=0.35$ associated with a closed model.

\section{THE QUINTESSENCE SCALAR POTENTIAL}

One of the possible modifications to the basic idea of quintessence includes the use of other potentials 16, 17, 18]. It may be interesting to investigate the situation in which the scalar potential $V(\chi)$ is of the form [19]

$$
V(\chi)=\alpha \dot{\chi}^{2}+\gamma
$$

where $\alpha$ and $\gamma$ are two constants. We may write $\gamma$ as $\gamma=\left(\alpha_{-}-\alpha_{+} w_{\chi}\right) \rho_{\chi}^{0}$, where $\alpha_{ \pm}=\frac{1}{2} \pm \alpha$.

Combining Eq. (27) with the evolution Equation for the field $\chi$ we get $\dot{\chi}$ as a function of the scale factor $a$, and thus we get

$$
\begin{array}{r}
\rho_{\chi}(a)=\rho_{\chi}^{0}\left[\alpha_{+}\left(\frac{a_{0}}{a}\right)^{3 / \alpha_{+}}+\alpha_{-}\right] \\
+\alpha_{+} P_{\chi}^{0}\left[\left(\frac{a_{0}}{a}\right)^{3 / \alpha_{+}}-1\right],
\end{array}
$$

and

$$
\begin{array}{r}
P_{\chi}(a)=P_{\chi}^{0}\left[\alpha_{-}\left(\frac{a_{0}}{a}\right)^{3 / \alpha_{+}}+\alpha_{+}\right] \\
+\alpha_{-} \rho_{\chi}^{0}\left[\left(\frac{a_{0}}{a}\right)^{3 / \alpha_{+}}-1\right],
\end{array}
$$

where the constants $\rho_{\chi}^{0}$ and $P_{\chi}^{0}$ represent the actual energy density and pressure, respectively, and are related to 
an arbitrary integration constant. From these two latter relations we get

$$
w_{\chi}(a)=\frac{w_{\chi}\left[\alpha_{-}\left(\frac{a_{0}}{a}\right)^{\frac{3}{\alpha_{+}}}+\alpha_{+}\right]+\alpha_{-}\left[\left(\frac{a_{0}}{a}\right)^{\frac{3}{\alpha_{+}}}-1\right]}{\left[\alpha_{+}\left(\frac{a_{0}}{a}\right)^{\frac{3}{\alpha_{+}}}+\alpha_{-}\right]+w_{\chi} \alpha_{+}\left[\left(\frac{a_{0}}{a}\right)^{\frac{3}{\alpha_{+}}}-1\right]},
$$

where, just as before, we take $w_{\chi}=\frac{P_{\chi}^{0}}{\rho_{\chi}^{0}}$. Note that for $w_{\chi}=\frac{\alpha_{-}}{\alpha_{+}}$we get $w_{\chi}(a)=w_{\chi}=$ const. This case corresponds to use $\gamma=0$ in Eq. (27). As we will soon see, this case allows us to write down an explicit expression for the quintessence scalar potential, $V(\chi)$. Through this paper, we will address this case only.

We could solve the time-time Einstein Equation (13) to obtain in this case

$$
\begin{aligned}
t=\frac{1}{H_{0}} & \frac{2}{\sqrt{\Omega_{M}}}\left(\frac{a}{a_{0}}\right)^{1 / 2} \\
& \times{ }_{2} F_{1}\left(\frac{1}{2}, \frac{\alpha_{+}}{\alpha_{-}} ; \frac{5}{6} ;-\frac{\Omega_{\chi}}{\Omega_{M}}\left(\frac{a}{a_{0}}\right)^{3 \alpha_{-} / \alpha_{+}}\right) .
\end{aligned}
$$

The quintessence scalar field $\chi$ in terms of the scale factor is given by

$$
\begin{array}{r}
\chi(a)=\chi_{0}+\varepsilon \operatorname{arcsinh}\left[\delta \left(\frac{a}{a_{0}} \sqrt{1+\delta^{2}}\right.\right. \\
\left.\left.-\sqrt{1+\delta^{2}\left(\frac{a}{a_{0}}\right)^{2}}\right)\right],
\end{array}
$$

where $\varepsilon=\frac{2}{3 H_{0}\left|w_{\chi}\right|} \sqrt{\frac{2 V_{\chi}^{0}}{\Omega_{\chi}} \frac{1}{\alpha_{-}+\alpha_{+}}}$and $\delta=$ $\left(\frac{\Omega_{\chi}}{\Omega_{M}}\right)^{1 / 3\left|w_{\chi}\right|}$.

Eq. (32) allows us to write down an explicit expression for the dark energy scalar potential $V(\chi)$. We get

$$
V(\chi)=V_{\chi}^{0}\left\{\frac{\delta}{\sinh \left[\varepsilon^{-1}\left(\chi-\chi_{0}\right)+\operatorname{arcsinh}(\delta)\right]}\right\}^{3 / \alpha_{+}},
$$

where $V_{\chi}^{0}$ represents the actual value of this potential. The hyperbolic potential (33) has been used in the literature [19, 20, 21]. This potential was studied for getting tracker solution from the corresponding field equations.

One of the characteristics of the scalar field $Q$ is given by the equation state parameter $w_{Q}(a)$, which is given by

$$
w_{Q}(a)=\frac{\left(3 w_{Q}^{0}+1\right)\left(w_{\chi}+1\right) \alpha_{-}\left(\frac{a_{0}}{a}\right)^{3 / \alpha_{+}}+\left(w_{Q}^{0}-w_{\chi}\right)\left(\frac{a_{0}}{a}\right)^{2}}{\left(3 w_{Q}^{0}+1\right)\left(w_{\chi}+1\right) \alpha_{-}\left(\frac{a_{0}}{a}\right)^{3 / \alpha_{+}}-\left(w_{Q}^{0}+w_{\chi}\right)\left(\frac{a_{0}}{a}\right)^{2}},
$$

Here, the quantities $w_{Q}^{0}$ are defined by

$$
w_{Q}^{0}=\frac{w_{\chi} \Omega_{\chi}+\Omega_{k} / 3}{\Omega_{\chi}-\Omega_{k}}=\left(w_{\chi}+\frac{1}{3}\right) \frac{\Omega_{\chi}}{\Omega_{Q}}-\frac{1}{3},
$$

where we have used the relation $\Omega_{k}+\Omega_{Q}=\Omega_{\chi}$ in the latter expression.

With $x \equiv a / a_{0}$, the scalar potential associated to the $Q$ field is given by

$$
\begin{aligned}
V_{Q}(x)=\rho_{Q}^{0}\left[\left(\frac{\alpha_{+}+\alpha_{-}}{2}\right)\right. & \left(1+w_{\chi}\right) x^{-3 / \alpha_{+}} \\
- & \left.\frac{2}{3} \frac{\Omega_{k}}{\Omega_{M}} x^{-2}\right],
\end{aligned}
$$

and the corresponding scalar field $Q$ is expressed by means of the following integral

$$
Q(x)=\frac{\sqrt{\rho_{Q}^{0}}}{H_{0}} \int_{x}^{1} \frac{d z}{z}
$$

$$
\times \sqrt{\frac{\left(1+w_{\chi}\right) \frac{\Omega_{\chi}}{\Omega_{Q}} z^{-3 \alpha_{-} / \alpha_{+}}-\frac{2}{3} \frac{\Omega_{k}}{\Omega_{Q}} z}{1+\left(1+w_{\chi}\right) \frac{\Omega_{\chi}}{\Omega_{M}} \alpha_{+} z^{-3 \alpha_{-} / \alpha_{+}}} .}
$$

A numerical integration shows that this potential presents the same characteristic than that described in the previous case, i.e. $V(Q)$ decreases when $Q$ increases, reaching the limit $V(Q) \longrightarrow 0$ for $Q \longrightarrow \infty$.

Finally, one interesting parameter to determine in this kind of model is the deceleration parameter, which is defined by $q=-\frac{\ddot{a}}{\dot{a} H^{2}}$, and when evaluated to present time, it becomes

$$
q_{0}=\frac{1}{2} \Omega_{M}-\frac{1}{2}\left(3\left|w_{\chi}\right|-1\right)\left(\Omega_{k}+\Omega_{Q}\right) .
$$

For $w_{\chi}<-1 / 3\left(1+\Omega_{M} / \Omega_{\chi}\right)$, the parameter $q_{0}$ becomes negative, in agreement with the observed acceleration of the universe. 


\section{CONCLUSIONS}

In this paper we have described a curvature universe model in which, apart from the usual CDM component, we have included a quintessence-like scalar field $Q$. We have fine-tuned the energy density, associated with this field, together with the curvature, for mimicking a flat model which resembles the quintessence (or dark energy) $\chi C D M$ model, which is characterized by $\Omega_{T}=\Omega_{M}+\Omega_{\chi}=1$. We have assumed for $Q$ an effective equation of state $P_{Q}=w_{Q} \rho_{Q}$, with $w_{Q}<0$. We have determined the form of the potential associated with this field, which effectively has the property of a quintessence potential, i.e. $V(Q)$ decreases when $Q$ increases, approaching zero asymptotically. Under the assumption that the equation state parameter was constant, we could describe explicitly the characteristics of the dark energy scalar field, $\chi$. Certainly, the basic idea of quintessence rests on the determination of the scalar potential as a function of this field.

Under an appropriate choice for the dark energy scalar potential, $V(\chi)$ (in terms of $\dot{\chi}$ ), we determine explicit ex- pression for this potential as a function of $\chi$. In this case we also gave expression for the scalar field $Q$ and its potential. Here, we determine the deceleration parameter which, under an appropriate choice of the parameters that characterize the model, became positive, in agreement with the acceleration detected by astronomical observations.

\section{Acknowledgments}

I am grateful to Francisco Vera for plotting assistance. Discussion with M. Cataldo, N. Cruz, S. Lepe, F. Peña and P. Salgado are acknowledged. I also acknowledge the Universidad de Concepción and Universidad del Bio-Bio for partial support of the Dichato Cosmological Meeting, where part of this work was done. This work was supported from COMISION NACIONAL DE CIENCIAS $\mathrm{Y}$ TECNOLOGIA through FONDECYT Projects $\mathrm{N}^{0}$ 1000305 and 1010485 grants, and from UCV-DGIP $\mathrm{N}^{0}$ 123.752 .
[1] S. White et al, Nature 366, 429 (1993).

[2] J. Mather et al, Astrophys. J., 420, L439 (1994).

[3] M. Roos and S. Harun-or-Rashid, The flatness of the Universe is Robust. Report No. astro-ph/0005541.

[4] P. De Bernardis et al, Nature 404, 955 (2000).

[5] S. Hanany et al, Astrophys. J., 545, L5 (2000).

[6] A. Guth, Phys. Rev. D, 23, 347 (1981).

[7] A. Riess et al, Astrophys. J., 116, 1009 (1998).

[8] S. Perlmutter et al, Nature 391, 51 (1998); Astrophys. J., 517, 565 (1999).

[9] R. R. Caldwell, R. Dave and P. Steinhardt, Phys. Rev. Lett. 80 , 82 (1998).

[10] E. W. Kolb, Astrophys. J. 344, 543 (1989).

[11] M. Kamionkowski and N. Toumbas, Phys. Rev. Lett., 77, 587 (1996).

[12] N. Cruz, S. del Campo and R. Herrera, Phys. Rev. D 58, 123504 (1998); M. Cataldo and S. del Campo, Phys. Rev. D 62, 025455 (2000); S. del Campo and N. Cruz, MNRAS, 317, 825 (2000).
[13] L. Wang, R. R. Caldwell, J. P. Ostriker and P. J. Steinhardt, Astrophys. J., 530, 17 (2000).

[14] T. D. Saini, S. Raychaudhury, V. Sahni and A. Starobinsky, Phys. Rev. Lett., 85, 1162 (2000).

[15] M. S. Turner, Time at the beginning. Report No. astro$\mathrm{ph} / 0106262$.

[16] A. Albrecht and C. Skordis, Phys. Rev. Lett., 81, 2076 (2000).

[17] P. Brox and J. Martin, Phys. Lett. B, 168, 40 (1999).

[18] I. Zlatev and P. J. Steinhardt, Phys. Lett. B, 159, 570 (1999).

[19] Y. Gong, quintessence model and observational constraints. Report No. gr-qc/0203007.

[20] T. Matos and L. Ureña-López, Phys.Rev.D 63, 063507 (2001).

[21] V. B. Johri, Search for tracker potential in quintessence theory. Report No. astro-ph/0108247. 